\title{
Seam Carving with Improved Edge Preservation
}

\author{
Johannes Kiess, Stephan Kopf, Benjamin Guthier, Wolfgang Effelsberg \\ University of Mannheim, 68131 Mannheim, Germany
}
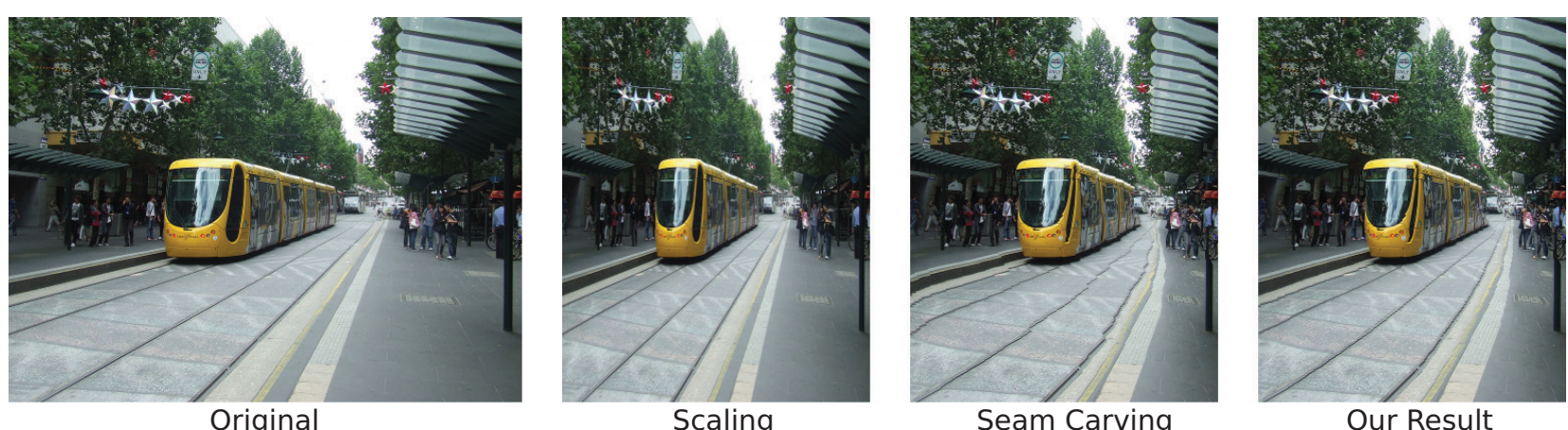

Figure 1. Our new algorithm compared to scaling and regular seam carving with forward energy. In this example, the width is reduced to $60 \%$ of the original size.

\begin{abstract}
In this paper, we propose a new method to adapt the resolution of images to the limited display resolution of mobile devices. We use the seam carving technique to identify and remove less relevant content in images. Seam carving achieves a high adaptation quality for landscape images and distortions caused by the removal of seams are very low compared to other techniques like scaling or cropping. However, if an image depicts objects with straight lines or regular patterns like buildings, the visual quality of the adapted images is much lower. Errors caused by seam carving are especially obvious if straight lines become curved or disconnected. In order to preserve straight lines, our algorithm applies line detection in addition to the normal energy function of seam carving. The energy in the local neighborhood of the intersection point of a seam and a straight line is increased to prevent other seams from removing adjacent pixels. We evaluate our improved seam carving algorithm and compare the results with regular seam carving. In case of landscape images with no straight lines, traditional seam carving and our enhanced approach lead to very similar results. However, in the case of objects with straight lines, the quality of our results is significantly better.
\end{abstract}

Keywords: seam carving, image retargeting, image adaptation, edge preservation

\section{INTRODUCTION}

The number of available high resolution images has increased considerably due to the technological progress of digital cameras in recent years. The improvement of the camera modules in mobile phones amplifies this development. Users do not only prefer to have access to these images on the Web or in digital archives via PC but also to take the images along on their mobile devices to share them with friends. One major limitation of mobile devices is that in most cases the resolution of digital images is significantly higher than the resolution of their displays. Additionally, the aspect ratios often differ which may lead to obvious distortions in large regions of the image.

Scaling the image or cropping its borders are the most frequently used methods to solve this task. Another widely employed technique which is normally used for the adaptation of wide screen movies to the resolution of television screens is to add black bars at the top and bottom of the image to get the desired aspect ratio

Further author information: (Send correspondence to)

Johannes Kiess: E-mail: kiess@informatik.uni-mannheim.de, Telephone: +49 (0)621 1812609

This is a preliminary version of an article published in

Proc. of IS\&T/SPIE Electronic Imaging (EI), Vol. 7542 (1), San Jose, CA, USA, January 2010

by Johannes Kiess, Stephan Kopf, Benjamin Guthier, Wolfgang Effelsberg 
(letterboxing). However, each of these techniques has its drawbacks and does either lead to obvious distortions, a loss of image content, or makes the image so small that it is difficult to perceive all the details.

A more efficient technique called seam carving was introduced by Avidan and Shamir. ${ }^{1}$ The idea is to remove image content which is less relevant for the understanding of the image. Seam carving works quite well for images without straight lines or regular patterns like landscape images but may cause distortions if used otherwise. Figure 1 shows an example in which seam carving causes noticeable deformations of objects: straight lines become curved or disconnected. Automatic ex post dewarping of the distortions is nearly impossible due to the large number of parameters and the high complexity of image warping algorithms. ${ }^{2}$

Our algorithm uses the seam carving technique and additionally preserves straight lines by preventing the removal of adjacent pixels which are located on such a line. Instead, the pixels chosen for the withdrawal are distributed all over the line. This allocation avoids bending or disconnecting of the line.

The rest of this paper is organized as follows. In Chapter 2, common image adaptation techniques and the typical limitations of existing methods are presented. In Chapter 3, we present the details of our new algorithm. Chapter 4 presents an evaluation and a comparison to regular seam carving. The paper concluded in Chapter 5 .

\section{RELATED WORK}

Image retargeting is a diverse field with a lot of different techniques available to resize an image. These approaches can be roughly divided into two categories: pixel removing and pixel merging. The first category contains all methods that remove pixels from an image in order to resize it, for instance cropping. Seam carving, which our new algorithm is based on, does also belong to this category. Methods from the second category reduce the image size by merging of pixels. Scaling is a typical and commonly used example for this kind of method.

\subsection{Pixel Removing}

Cropping is a common image adaptation technique which removes image content from the borders of an image. Suh et al. ${ }^{3}$ proposed a basic cropping approach for the automatic generation of thumbnails. The important regions of the image are identified and then the other parts of the image are cropped. Finally, the edited image is scaled to the target size. The authors use two different approaches to identify the Regions-of-Interest (ROI): The first one uses a saliency map ${ }^{4}$ and is applicable to all different kinds of images. The second one considers semantic information by using face detection and is therefore restricted to images with people.

Automatic browsing, a method presented by Liu et al., ${ }^{5}$ simulates the browsing a user has to do while looking at an image with high resolution on a device with a small display. The main idea is to show the important regions of the image like a slideshow one after another. Hence, the image is searched for Attention Objects (AO) by using saliency as well as face and object detection. Nearby AOs are then grouped to form ROIs. These ROIs are fitted into a rectangle of the target size by zooming. Each ROI additionally gets a value which represents the time a user needs to be able to grasp all the information shown. After that, a path over the source image is calculated which shows all ROIs consecutively for the computed time span. The path between two ROIs is always the shortest path between the two centers of the regions.

In previous work, we have presented an adaptation technique for mobile devices where we put the focus on a fast and efficient implementation. ${ }^{6}$ Cropping is used in a first step to remove border regions which do not contain relevant semantic content (e.g., sky). Attention objects like faces, text regions and contrast based saliency maps are identified in a second step and combined into a region of interest. We proposed a semi-automatic approach to correct obvious errors in the adapted images.

In our mobile cinema project, ${ }^{7}$ we have implemented an extension for videos, which automatically segments and classifies moving objects. ${ }^{8,9}$ Additional semantic features like people or moving objects improve the selection of a region. A heuristic approach is used to maximize the information that is visualized in the final video. ${ }^{10}$ Several constraints are considered like a minimum perceptible size and maximum reasonable size for features, and the preservation of the aspect ratio. ${ }^{11} \mathrm{~A}$ combination of cropping and scaling is used. To increase the overall content that is visualized, the selected region may change in time by adding an artificial zoom or camera pan. 
Seam carving, introduced by Avidan and Shamir, ${ }^{1}$ is the basis of our new algorithm. Therefore, it is handled in a separate subsection in more detail.

A general drawback of pixel removing is that some image regions are lost in the process. This may lead to missing or truncated objects. Hence, the main purpose of the methods in this category is to remove content with the lowest information value for the viewer.

\subsection{Pixel Merging}

The previously presented methods (based on the removal of pixels) discard image content which is not completely located in a ROI. To avoid this, Liu et al. ${ }^{12}$ developed a non-photorealistic fisheye-view warping method, which emphasizes the ROI and scales down the unimportant parts of an image. At first, an importance map is created which combines saliency with object detection like face detection. Based on this map, the ROI in the image is identified and defined as center of the target image, although it is not necessarily positioned in the middle. The ROI is scaled down until it takes up to $70 \%$ of the space of the target image. The rest of the image is scaled down based on two different methods. Radial warping scales every pixel in relation to its place between ROI and the border of the source image. The further away from the center, the smaller it becomes. Cartesian fisheye warping seperates the image into nine regions. The ROI again is the center of the new image and is surrounded by eight regions. Each is scaled uniformly and their size is determined based on their position between the ROI and the border on the source image.

Setlur et al. ${ }^{13}$ propose to protect the important objects of an image by cutting them out. They segment an image into regions by using the mean-shift algorithm. ${ }^{14}$ Then they combine adjacent regions based on their color and intensity. Additional relevant regions are identified by face detection and saliency. Should these regions be small enough to be contained in the target size, the image is simply cropped. Otherwise, these regions are removed and the background is repaired by inpainting. ${ }^{15}$ The background is then scaled to the target size and the removed objects are inserted. If the objects are too big to fit in, they are scaled in inverse proportion to their importance.

The warping method proposed by Wolf et al. ${ }^{16}$ is mainly designed for video retargeting but is also applicable to image retargeting. Each pixel is assigned an importance value based on a gradient-based saliency and face detection. The pixels are then mapped into an image of the target size. Unimportant pixels are mapped into a small region and merged with their neighbors, while important pixels are mapped into a region of similar size. This preserves important regions at their original size if possible.

Optimized Scale-and-Stretch by Wang et al. ${ }^{17}$ partitions the source image into small regions and determines an optimal scaling factor for each of the regions. This allocation is achieved by laying a mesh over the source image. A significance map, which is created based on a gradient and a saliency map, marks the regions with higher importance. These regions are scaled uniformly, while the other regions are allowed to be more distorted. Deformation energy and grid line bending energy are introduced and minimized to prevent major distortions of important objects and structures which span over several regions.

Guo et al. ${ }^{18}$ also use a mesh for their image retargeting approach, but instead of using quadratic cells they use triangular cells and formulate the retargeting as a mesh parametrization problem. Considering important regions of the image based on saliency and face detection, a controlling mesh is constructed that is consistent with the underlying image structures. This is done by using feature points to build the mesh. The saliency information is then associated with the mesh. Three constraints - the boundary constraint, the saliency constraint, and the structure constraint - are used to preserve salient objects and structures.

The methods in this category try to shift major distortions to non-relevant image regions where they are not so obvious to the viewer. If no such region is available in the image, the result becomes either distorted or similar to conventional scaling. In addition, many of the algorithms have problems with preserving distinctive image lines. 

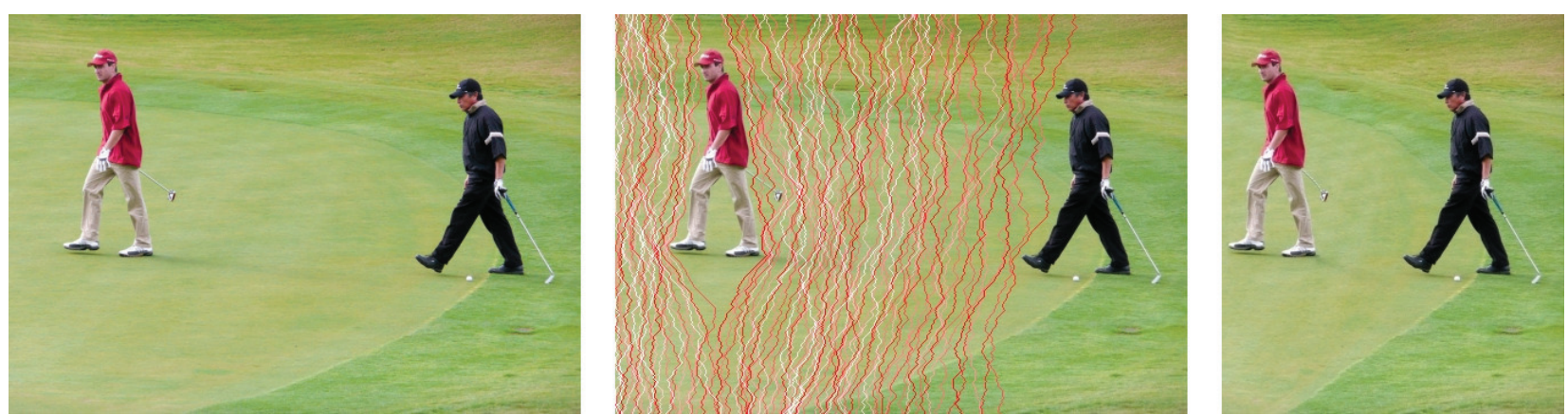

Figure 2. Left: Original image. Center: The color indicates the order of the seams (white seams with low energy are removed first, red seams are preserved as long as possible). Right: Image after the seams have been removed.

\subsection{Seam Carving}

Seam Carving is a technique proposed by Avidan and Shamir ${ }^{1}$ for content-aware resizing of images. This technique belongs to the category of pixel removing. The basic idea is to remove paths of low energy pixels (seams) from top to bottom or from left to right which are not so important for the understanding of the image content.

The removal of each seam causes a reduction of the image size by one where vertical seams reduce the width and horizontal seams reduce the height. Figure 2 shows an example with vertical seams and the final image after removing them.

A vertical seam $\mathbf{s}=\{(x(i), i)\}_{i=1}^{H}$ in an image $I$ with height $H$ is subject to two conditions in order to be valid. First, the seam consists of one and only one pixel in each row, and second the horizontal distance between two adjacent seam pixels $|x(i)-x(i-1)|$ is not allowed to exceed a threshold of $T$. In case of $T=1$, the seam pixels of vertical seams are vertically or diagonally connected (8-connected). These conditions lead to the following definition of a vertical seam:

$$
\mathbf{s}=\left\{s_{i}\right\}_{i=1}^{H}=\{(x(i), i)\}_{i=1}^{H}, \text { s.t. } \forall i:|x(i)-x(i-1)| \leq T
$$

The pixels of a seam are chosen based on an energy function $e$, whereas the authors suggest to use the $e_{1}$ error norm:

$$
\mathbf{e}_{\mathbf{1}}(\mathbf{I})=\left|\frac{\partial}{\partial x} I\right|+\left|\frac{\partial}{\partial_{y}} I\right|
$$

Other recent work proposes to use wavelet decomposition ${ }^{19}$ as energy function. Additional information may modify the energy function like a human attention model based on face detection and saliency. ${ }^{20}$

Dynamic programming is used to identify the optimal seam $s^{*}$, which is defined as the seam with the minimum cost based on the energy function $e$. The cost of a seam is the sum of the energy values of all path pixels. To calculate these costs for a vertical seam, the cumulative minimum energy $M$ for all pixels $(i, j)$ is computed by traversing the image from the first row to the last row.

$$
\mathbf{M}(\mathbf{i}, \mathbf{j})=e(i, j)+\min (M(i-1, j-1), M(i-1, j), M(i-1, j+1))
$$

The minimum value of $M$ in the last row indicates the total cost of a seam with the lowest energy. By backtracking from this minimum, we find the path of the optimal seam. The computation of $M$ for horizontal seams is equivalent.

If both dimensions of an image should be changed, it is necessary to build the optimal order of vertical and horizontal seams. Therefore, the minimum energy of vertical and horizontal seams is compared and the lower one is chosen. This is repeated and an optimal order is built. 
Rubinstein et al. ${ }^{21}$ enhance the seam carving algorithm by introducing a new energy criterion as well as the use of graph cuts instead of dynamic programming. The improved method is also capable of retargeting videos.

Seam carving for images can be formulated as a minimum cost graph cut problem. A grid-like graph is constructed in which each node represents a pixel of the image and is connected to each of its neighboring pixels. All the pixels in the leftmost and rightmost columns are connected to two virtual terminal nodes $S$ (source) and $T$ (sink) with infinite weight arcs. The optimal seam is defined as $S / T$ cut (or simply cut) with the minimum costs among all valid cuts. The pixels of the optimal seam are the ones on the left side of the cut.

Two additional constraints are required in order to get a valid seam: monotonicity and connectivity. These constraints are achieved by using different weights for the different directions of the arcs.

For video retargeting, the grid is extended by adding time as the third dimension. All left and right nodes in the frames are connected to the source and the sink nodes respectively and the same constraints as above have to be maintained between adjacent frames.

In the original seam carving approach, the seams with the lowest energy costs were removed. However, this may lead to distortions and artifacts because it ignores the energy that is brought into the image by the new edges that result from the pixel removal. The new energy criterion proposed by the authors looks forward in time and determines the energy value of each pixel based on these new edges.

In order to overcome some of the limitations of seam carving, such as structured backgrounds, Rubinstein et $a .^{22}$ proposed multi-operator media retargeting, which combines the three different resizing operators scaling, cropping, and seam carving. An image similarity measurement called bi-directional warping is introduced to determine in which order the operators are used. This order is searched via dynamic programming in the resizing space, which is defined as a three dimensional space to combine the operators. The final path can either be a regular path, where each operator is only used once, or a mixed path, where each operator is used as often as necessary.

In previous work, we used seam carving to adapt videos to different screen resolutions. ${ }^{23}$ We proposed a fast technique where the optimization problem to find optimal seams (seam manifolds) is transformed from 3D into $2 \mathrm{D}$ space. At first, a background image is generated for each shot. Seams are then detected in the background image and transferred back into each frame. Only valid seams that are included in all frames of a shot are used for size adaptation. The advantage of the computationally intensive graph cuts technique ${ }^{21}$ compared to our approach is the fact that the visual quality of graph cuts is slightly better in some videos.

\section{IMPROVED SEAM CARVING}

Errors caused by seam carving are especially disturbing when the original image includes regular patterns or straight lines. These lines may become curved or disconnected in the final image. Figure 3 shows an example of an image with straight lines which has been adapted through seam carving. The curved lines on the windmill vane are clearly visible. These distortions may occur if a seam crosses a non-vertical or non-horizontal line (see Figure 4).

When, for instance, a vertical seam is removed from an image, all the pixels on the right side of the seam are moved one pixel to the left in order to close the gap. If this is repeated and several seams are crossing a straight line in adjacent intersection points, a distortion becomes visible when the pixels are moved up (see Figure 4 c)).

\subsection{Workflow of the algorithm}

In most images, an optimal seam crosses one or more objects (e.g., buildings, streets, tree trunks, cars, street lights) and deforms straight lines. It is not our goal to shift an optimal seam so that the seam does not cross an object. By using a different energy map, we rather change some optimal seams so that the visible effect of errors is reduced and the viewer does not notice the distortion. It should be guaranteed that seams do not cross a straight line in adjacent pixel positions. If the intersection points are scattered all over the line, the distortion is distributed and not so obvious to the viewer. 


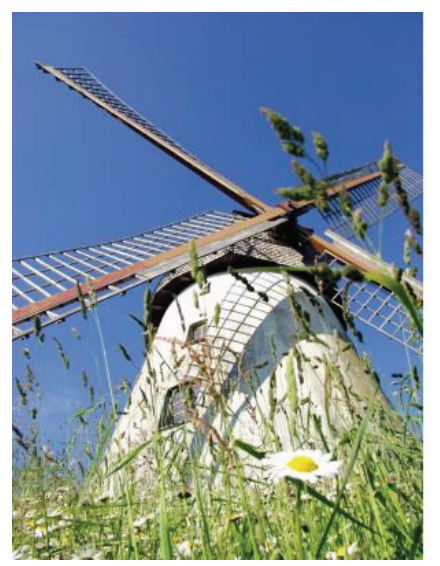

Figure 3. Left side: original image. Right side: image adapted to $75 \%$ of the original width using seam carving (forward energy used). Noticeable curved lines are marked with red rectangles.

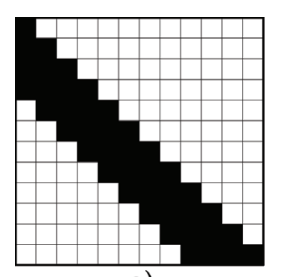

a)

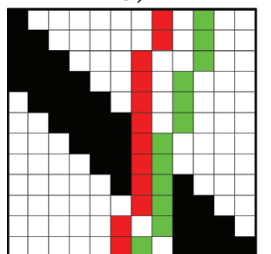

b)

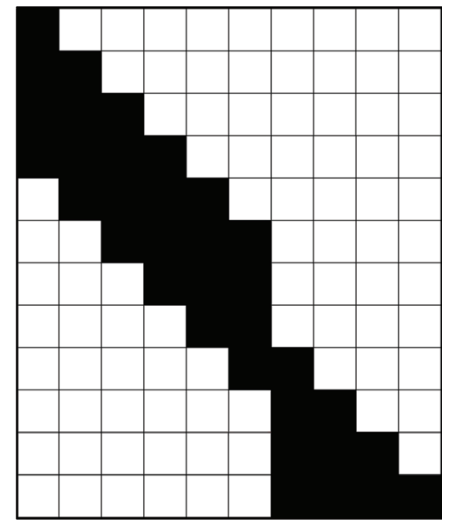

c)

Figure 4. a) A straight line. b) Two seams crossing the line. c) An obvious distortion occurs by removing adjacent seams.

We apply an automatic line detection algorithm to identify straight lines in images. Each time a seam crosses a line, the energy in a certain radius of the intersection point is increased in order to reduce the probability that other seams cross the line in adjacent pixel positions.

Our algorithm is based on the regular seam carving algorithm and therefore some of the basic steps are similar. In addition, we use the information about the most relevant straight lines. We apply the Canny edge detector $^{24}$ to identify significant edges. Afterwards, the edge pixels are transformed into Hough space ${ }^{25}$ to locate pixels on straight lines. Edge pixels that are located on straight lines will be called line pixels in the following.

The only enhancement required for seam carving is to change the computation of the energy map. For each selected optimal seam, the intersection points of optimal seams and lines pixels are detected, and the energy map is modified in the local neighborhood of all these points. Figure 5 gives an overview of the different steps of the algorithm. The details of the line detection algorithm and the improvements of seam carving are presented in the following sections.

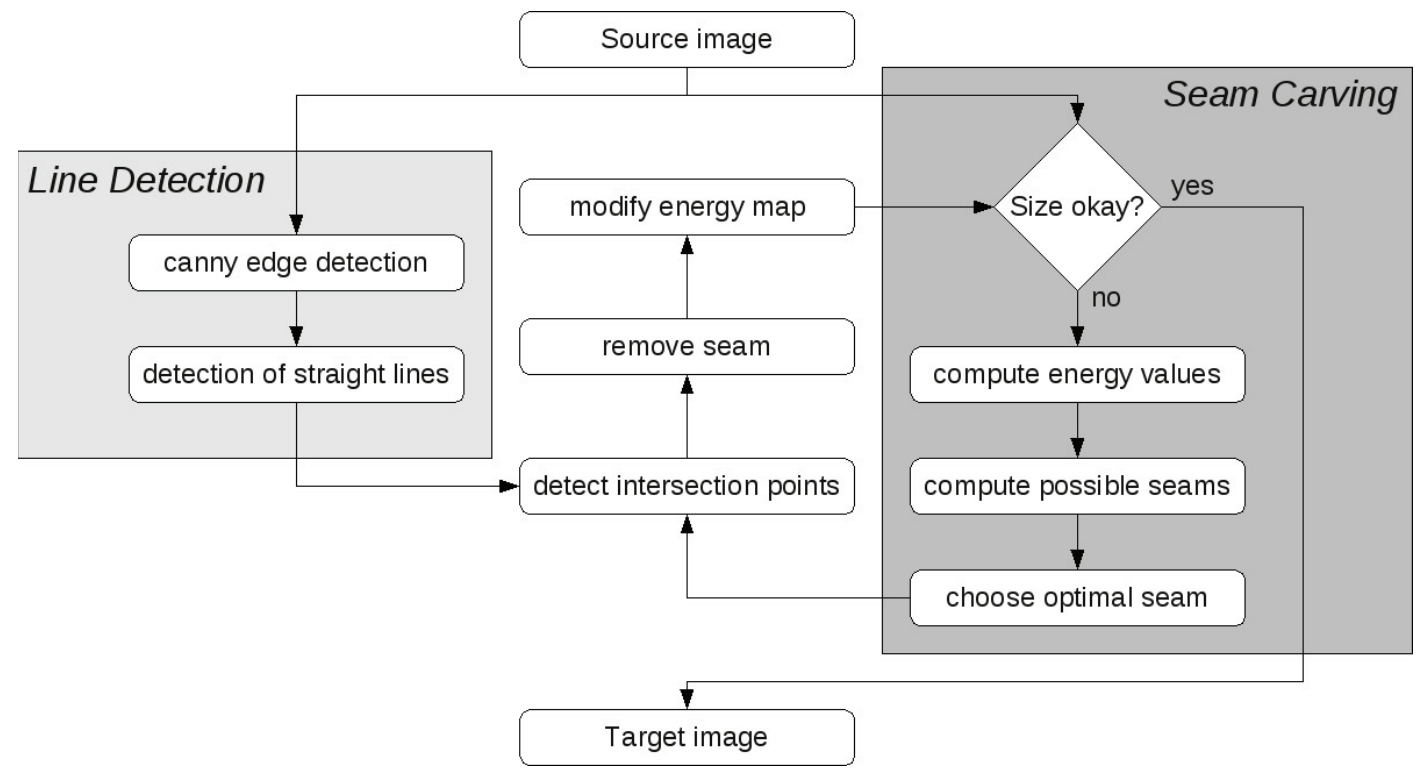

Figure 5. Overview of the workflow of the algorithm 

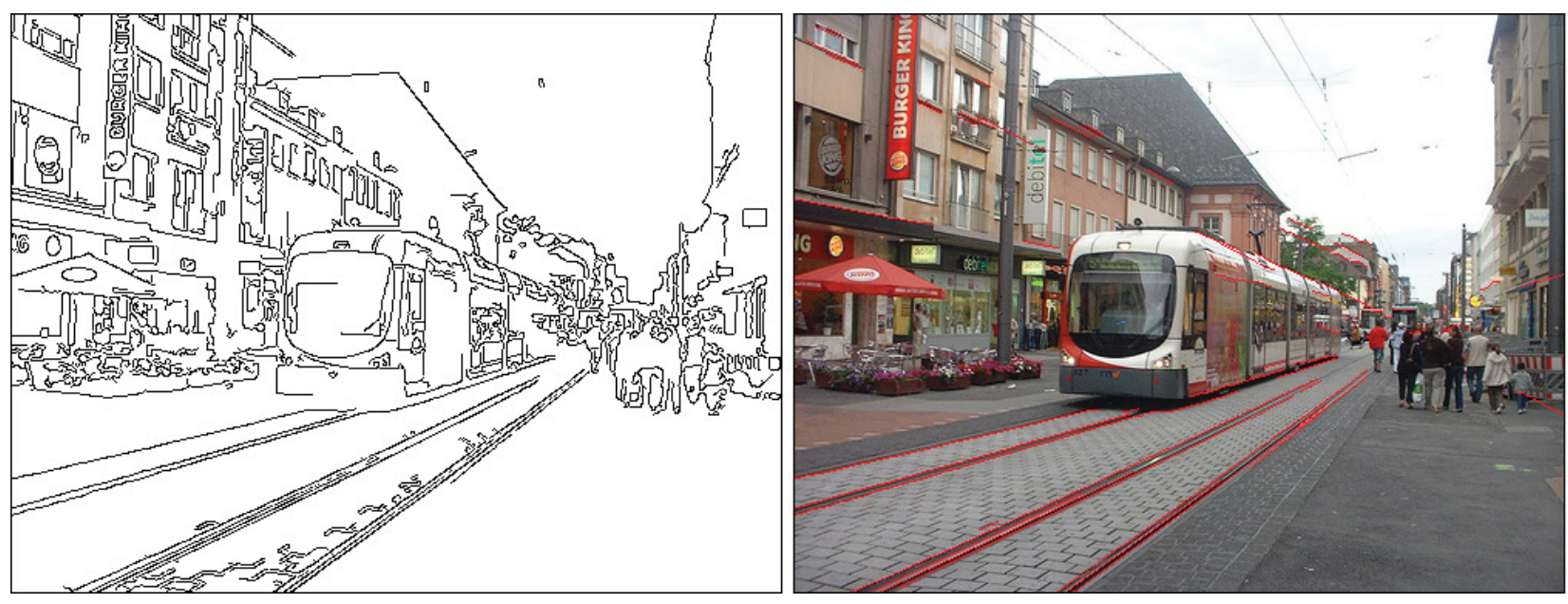

Figure 6. Edge image and detected lines (red)

\subsection{Detection of straight lines}

Edges in the image are detected based on the Canny edge detector. As parameters of Canny, we use a Gaussian mask of size 3 for noise reduction, and $T_{u p}=100$ and $T_{l o w}=20$ as upper and lower thresholds for the hysteresis.

Edge pixels are transformed into Hough space $I_{H}$ next. Each point in Hough space corresponds to a straight line in the edge image. A threshold $T_{\text {hough }}=0.6 \cdot \max \left\{I_{H}\right\}$ is derived from the maximum value in Hough space. Only the most significant straight lines are selected by considering Hough pixels that exceed this threshold.

For each line candidate, the number of edge pixels located on this line is is counted. An edge pixel is considered as line pixel, if the distance between edge pixel and line is below a threshold $T_{\text {dist }}=0.5$ pixels, and if the line segment has a length of at least $T_{\text {length }}=10$ pixels. Small gaps between valid line segments are filled up $\left(T_{\text {gap }}=30\right)$. Because the precision of the detected lines is not sufficient, we use a gradient descent algorithm to optimize the parameters of a line by maximizing the total number of line pixels on each line. Figure 6 shows an example of an edge image and the straight lines that are detected automatically.

\subsection{Modification of the energy map}

The seam carving algorithm starts by computing an energy value for each pixel based on the gradient. We also use forward energy as proposed by Rubinstein. ${ }^{21}$ An additional energy map that adds an offset value to each gradient is initialized with zero. All seams are calculated based on the energy values and the optimal seam is selected.

Along the path of the optimal seam, the intersection points of the seam and the detected lines are marked. The offset energy map is increased in the local neighborhood of these points. By calculating the next optimal seam, the values of the offset energy map are added to the gradient values. This guarantees that seams avoid intersection points in the following iterations. Figure 7 visualizes the modification of the energy map.

The intersection point of the offset energy map is increased by a value of 200, and adjacent pixels in a region of $7 \times 7$ pixels are increased according to a $2 \mathrm{D}$ Gaussian distribution. After the modification of the offset energy map, the pixels of the optimal seam are removed from the image and the offset energy map. The algorithm stops after a sufficient number of seams have been removed to reach the target image size.

\section{EVALUATION}

In the following, we evaluate our enhanced seam carving algorithm and compare the quality of the adapted images to the quality of regular seam carving based on forward energy. In case of landscape images where no straight lines are detected, traditional seam carving and our enhanced approach lead to identical results. On the other hand, when the image contains objects with straight borders, the quality of the adapted image is 

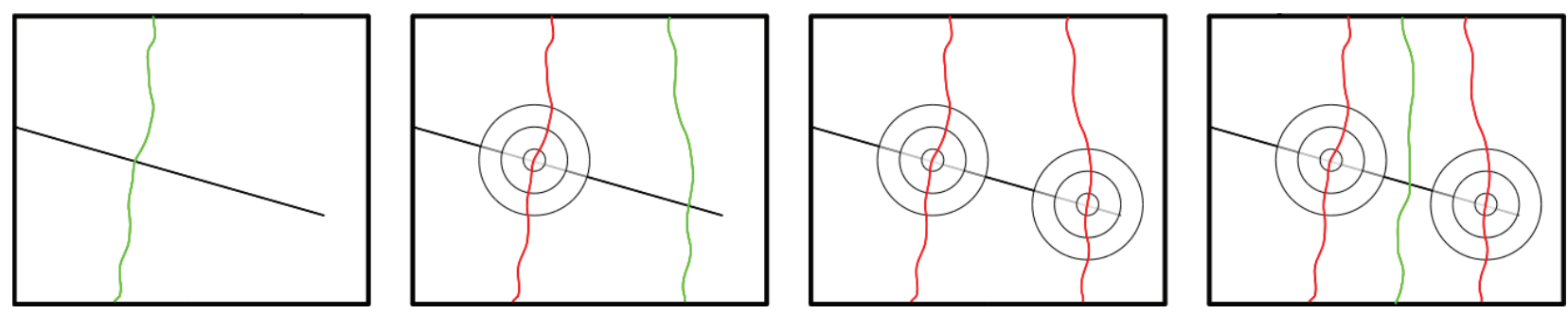

Figure 7. Visualization of the basic idea of the modification of the energy map: The optimal seam (green) of one iteration may cross a straight line (black). After the removal of the seam (the removed seam is marked in red), the energy in the local neighborhood of the intersection point is increased. This decreases the probability that an optimal seam crosses these areas in the next iterations.

significantly better. Figure 8 compares the original seam carving with our enhanced approach. Most critical for seam carving are diagonal lines. The red circles mark distorted lines in the adapted images of regular seam carving.

The first two image rows contain images which show diagonal straight lines reaching from one side of the image to the other. This kind of images is problematic for seam carving because it can not avoid to cross these lines. The bridge in the first row becomes curved because seam carving accumulates the seams on the left side of the image due to large areas of water and sky. The algorithm does not take lines into account and removes these regions. The result of seam carving in the second row shows blurred and bent lines in most parts of the image. Our method distributes the seams evenly across the lines and reduces these errors. Although straight lines also become slightly curved in some cases, the visual distortion caused by the enhanced seam carving is much less obvious.

Many objects like trees or people are depicted in the image of the third row, which makes it difficult to remove seam pixels and maintain these objects at the same time. Although the most relevant straight lines do not reach over the entire image, they are located in an area with less relevant content and thus become blurred.

The image in the last row is similar to the first two images based on straight lines spanning over the image but depicts a landscape with no structured background. The adapted image based on seam carving has blurred and broken lines on the left and the right side, while the other regions of the image show no distortions. Again, our method overcomes these distortions and achieves a higher visual quality.

An example of the limitations of our approach is shown in Figure 9. The pedestrian underpass contains a lot of straight lines which cover all regions of the image. The algorithm can only prevent straight lines if there is sufficient space to move the seams. In this example, the lines are too close to each other and the angles between the lines differ, so that it is not possible to preserve one line without distorting another. If a large number of straight lines or straight structures are contained in an image, the algorithm may not be able to prevent them from bending or getting distorted.

\section{CONCLUSIONS AND OUTLOOK}

We presented an algorithm for image retargeting and put our focus on the preservation of straight lines. Our algorithm is based on the seam carving algorithm and additionaly adds line detection and preservation. When a seam crosses a straight line, adjacent energy values are increased in order to prevent the following seams from crossing the line nearby. The distribution of the seams preserves straight lines much better and less distortions are included in the adapted image. Compared to the original seam carving, our method achieves significantly better results when used on images with prominent straight lines or structures.

In future work, we would like to extend our line preserving approach to the retargeting of videos, where similiar problems occur. Another possibility would be to integrate and combine our method with other popular image retargeting approaches like image warping. 

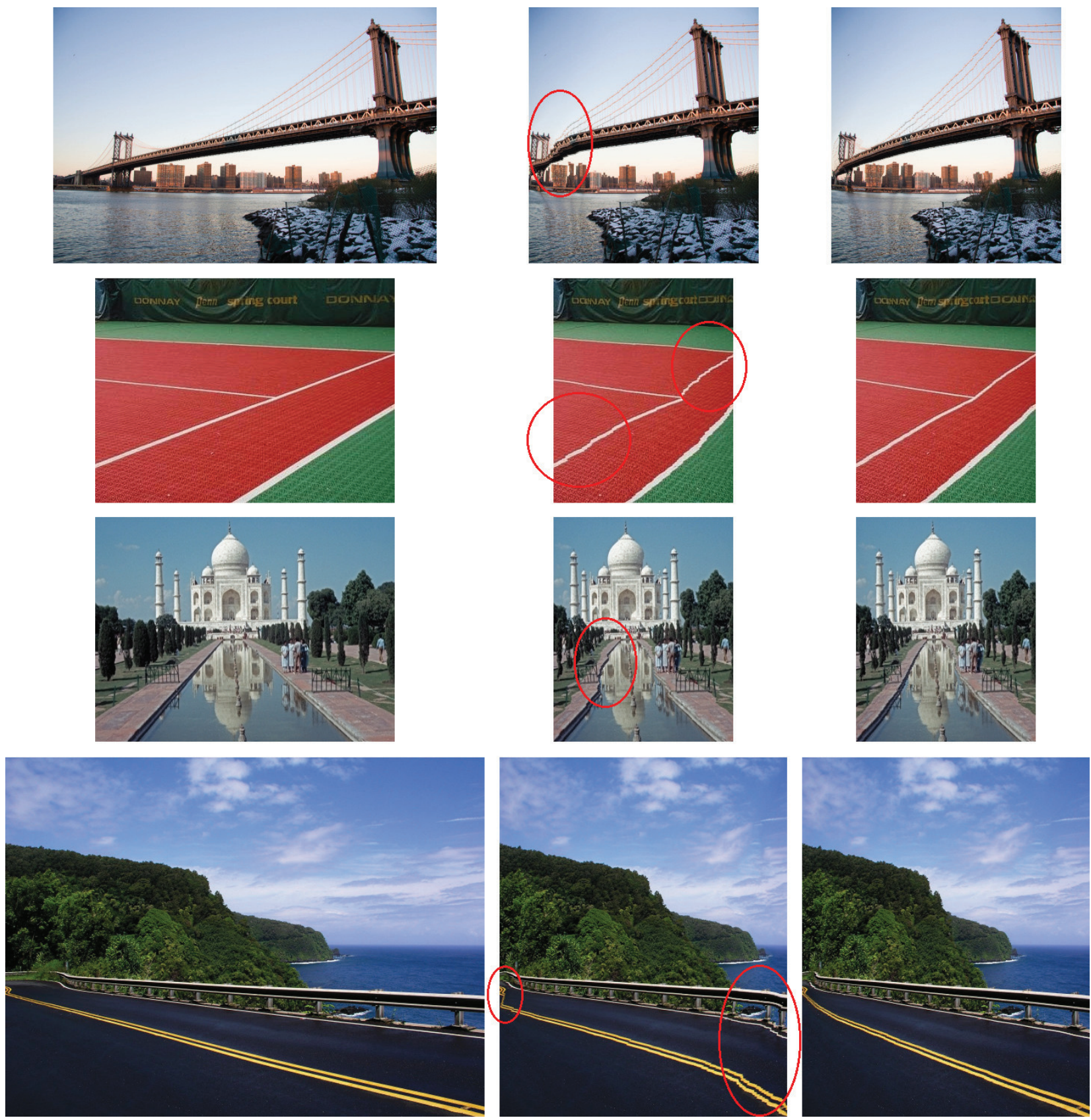

Figure 8. Left: Original. Center: Seam carving with forward energy. Right: Our result. All the images have been reduced to $60 \%$ of their original width. The red circles mark distorted lines in the seam carving results. 

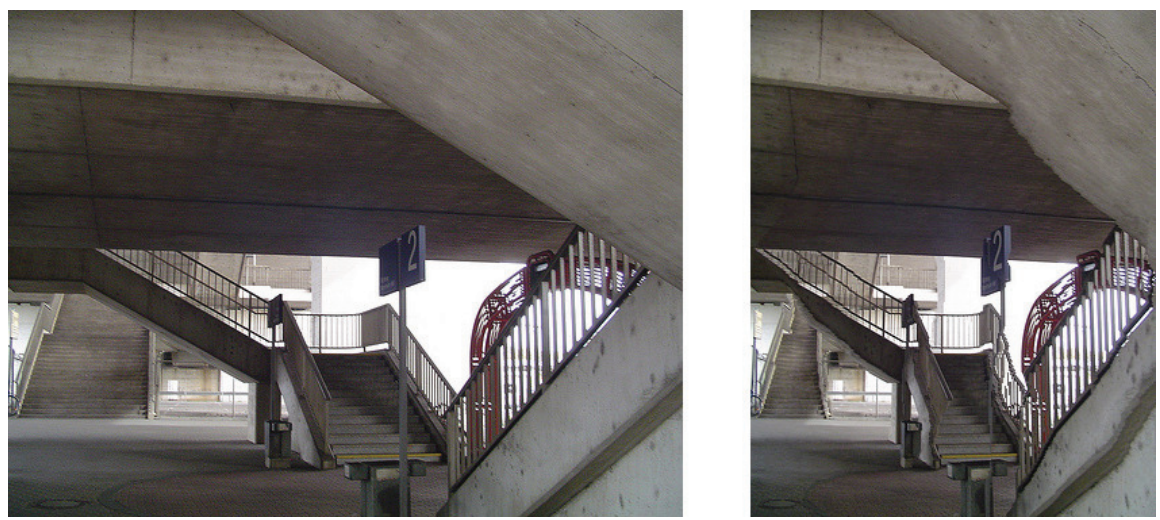

Figure 9. Limitations of the algorithm

\section{Acknowledgements}

We want to thank the following flickr.com users for providing their images via the creative commons license: Matthew Black (tram.jpg), rioncm (golf.jpg), teoruiz (bridge.jpg), the_tahoe_guy (road.jpg) and UweF (pedestrian_underpass.jpg). Furthermore, the authors acknowledge the financial support granted by the Deutsche Forschungsgemeinschaft (DFG).

\section{REFERENCES}

[1] Avidan, S. and Shamir, A., "Seam carving for content-aware image resizing," ACM Transactions on Graphics, SIGGRAPH $2007 \mathbf{2 6}$ (3) (2007).

[2] Haenselmann, T., Busse, M., Kopf, S., King, T., and Effelsberg, W., "Multi perspective panoramic imaging," Elsevier Image and Vision Computing Journal 27 (4), 391 - 401 (March 2009).

[3] Suh, B., Ling, H., Bederson, B., and Jacobs, D., "Automatic thumbnail cropping and its effectiveness," in [Proceedings of the 16th annual ACM symposium on User interface software and technology], 95-104 (2003).

[4] Itti, L., Koch, C., and Niebur, E., "A model of saliency-based visual attention for rapid scene analysis," IEEE Transactions on Pattern Analysis and Machine Intelligence 20(11), 1254-1259 (1999).

[5] Liu, H., Xie, X., Ma, W.-Y., and Zhang, H.-J., "Automatic browsing of large pictures on mobile devices," in [ACM international conference on Multimedia], 148-155, ACM Press (2003).

[6] Kopf, S., Guthier, B., Lemelson, H., and Effelsberg, W., "Adaptation of web pages and images for mobile applications," in [Proceedings of ISETT/SPIE conference on Multimedia on Mobile Devices], 7256, 72560C72560C-12 (2009).

[7] Kopf, S. and Effelsberg, W., "Mobile cinema: Canonical processes for video adaptation," in [Multimedia Systems], 14(6), 369-375, Springer (December 2008).

[8] Farin, D., Haenselmann, T., Kopf, S., Kühne, G., and Effelsberg, W., "Segmentation and classification of moving video objects," in [Handbook of Video Databases: Design and Applications], Furht, B. and Marques, O., eds., Internet and Communications Series 8, 561-591, CRC Press, Boca Raton, FL, USA (September 2003).

[9] Kopf, S., Haenselmann, T., and Effelsberg, W., "Enhancing curvature scale space features for robust shape classification," in [Proceedings of IEEE International Conference on Multimedia and Expo (ICME)], 478481, IEEE Computer Society Press (July 2005).

[10] Kopf, S., Lampi, F., King, T., and Effelsberg, W., "Automatic scaling and cropping of videos for devices with limited screen resolution," in [ACM Multimedia (video program session)], 957-958 (October 2006).

[11] Kopf, S., Haenselmann, T., Farin, D., and Effelsberg, W., "Automatic generation of summaries for the web," in [Proceedings of ISETT/SPIE conference on Storage and Retrieval for Media Databases], 5307, 417-428 (January 2004).

[12] Liu, F. and Gleicher, M., "Automatic image retargeting with fisheye-view warping," in [Proceedings of the 16th annual ACM symposium on User interface software and technology], 153-162 (2003). 
[13] Setlur, V., Takagi, S., Raskar, R., Gleicher, M., and Gooch, B., "Automatic image retargeting," in [Proceedings of the 4th international conference on Mobile and ubiquitous multimedia], 247-250 (2005).

[14] Comaniciu, D. and Meer, P., "Mean shift: A robust approach towards feature space analysis," IEEE Transactions on Pattern Analysis and Machine Intelligence 24, 603-619 (2002).

[15] Harrison, P., "A non-hierarchical procedure for re-synthesis of complex textures," in [The 9th International Conference in Central Europe on Computer Graphics, Visualization and Computer Vision], 190-197 (2001).

[16] Wolf, L., Guttmann, M., and Cohen-Or, D., "Non-homogeneous content-driven video-retargeting," in [Proceedings of the Eleventh IEEE International Conference on Computer Vision (ICCV-07)], (2007).

[17] Wang, Y.-S., Tai, C.-L., Sorkine, O., and Lee, T.-Y., "Optimized scale-and-stretch for image resizing," $A C M$ Trans. Graph. 27(5), 1-8 (2008).

[18] Guo, Y., Liu, F., Zhou, Z.-H., and Gleicher, M., "Image retargeting using mesh parameterization," IEEE Transactions on Multimedia 11(5), 856-867 (2009).

[19] Han, J.-W., Choi, K.-S., Wang, T.-S., Cheon, S.-H., and Ko, S.-J., "Improved seam carving using a modified energy function based on wavelet decomposition," in [IEEE 13th International Symposium on Consumer Electronics], 38-41 (May 2009).

[20] Hwang, D.-S. and Chien, S.-Y., "Content-aware image resizing using perceptual seam carving with human atention model," in [IEEE International Conference on Multimedia and Expo], 1029-1032 (June 2008).

[21] Rubinstein, M., Avidan, S., and Shamir, A., "Improved seam carving for video retargeting," ACM Transactions on Graphics, SIGGRAPH $200827(3)$ (2008).

[22] Rubinstein, M., Shamir, A., and Avidan, S., "Multi-operator media retargeting," ACM Transactions on Graphics, SIGGRAPH 2009 28(3), 1-11 (2009).

[23] Kopf, S., Kiess, J., Lemelson, H., and Effelsberg, W., "Fscav: fast seam carving for size adaptation of videos," in [MM '09: Proceedings of the Seventeenth ACM international conference on Multimedia], 321330, ACM, New York, NY, USA (2009).

[24] Canny, J. F., "A computational approach to edge detection," in [IEEE Transactions on Pattern Analysis and Machine Intelligence], 8(6), 679-698, IEEE Computer Society Press (1986).

[25] Duda, R. O. and Hart, P. E., "Use of the hough transformation to detect lines and curves in pictures," Commun. ACM 15(1), 11-15 (1972). 